\title{
Construction of a Three-sided Immersive Telecollaboration System
}

\author{
Christian P. Spagno Andreas M. Kunz \\ Swiss Federal Institute of Technology Zurich \\ Center for Product Development \\ cspagno@imes.mavt.ethz.ch \\ kunz@imes.mavt.ethz.ch
}

\begin{abstract}
The so-called "blue-c" is a novel three sided immersive projection system, which has been especially designed to support telecollaborative teamwork [4]. Therefore, special projection screens are used, which can be switched to a transparent state electrically. The user is captured with sixteen cameras in total, standing outside of the projection room. These images are then processed to create a $3 D$ representation of the user, which can be projected in a second installation. The whole installation has been engineered and implemented at the ETH Zurich [9]. A special active stereo projection system, based on LCD projectors with additional shutters proved to work very well at a very competing price. The paper describes the background of the decisions made and the problems that had to be overcome.
\end{abstract}

\section{Introduction}

Immersive visualization of computer data is a key feature of most Virtual Reality systems. Many immersive VR systems are based on stereo projection technologies. The systems may vary from an inexpensive single wall projection setup to a sophisticated multi wall active stereo projection installation. The projector itself is just one component of a VR installation and together with the projection screens maybe one of the most important parts. However, other components like cables. splitters and projector mountings do also have a big impact on the overall performance of the system. In addition further components like tracking system and loudspeakers do also influence the projection system and thus the overall immersion.

The blue-c system realized at the ETH Zurich is a three sided projection installation. In addition to the immersive projection capabilities, the blue-c integrates some special features like liquid crystal projection screens to enable picture acquisition through the screen with totally 16 cameras. The images of the 16 cameras are processed in real time [8], [12]. A 3D representation of the blue-c user is generated from these images and then displayed together with other virtual objects in a second VR installation, allowing new ways of telecollaboration [13], [15].

Since new technologies and new materials were combined to a new system, some components had to be custom made in order to support the simultaneous picture acquisition and stereo projection. But even the design of the "classical" projection part was quite time consuming, particularly in the case of a limited budget. Many immersive projection system like the CAVE [1] and ReaCTor [6] have been successfully set up, but even though there are many open questions when a new system has to be built and decisions have to be made. The goal of this paper is to show how the blue-c was set up and to discuss the first experiences with the new system. This paper will only address the projection and acquisition system of the blue-c, including the mechanical and electrical installations, including the problems that arise and the background of the decisions that have been made.

\section{The blue-c}

The blue-c is based on a three-sided stereo projection system. In order to have a full coverage of the user inside the system, 16 cameras are placed outside of the projection room (Fig. 1).

Special liquid crystal projection screens allow the cameras to look inside the projection room while quasisimultaneously serving as a back projection screen for the user. This LC projection screens can be electrically switched from an opaque state to a transparent state. In the blue-c they are switched between this two states 60 times per second, allowing a time multiplexing between projection and picture acquisition [5]. During each opaque state an image for the left and an image for the right eye are projected with an active stereo projection system. During the transparent phase the cameras take a picture of the user inside the system. In addition an active illumination is triggered and the projection output is blocked to enhance the acquired image quality. In order to protect the user's eyes from the active illumination flash, the shutter glasses of the active stereo system are modified 
electronically with a third step where both eyes are darkened [2], [3].

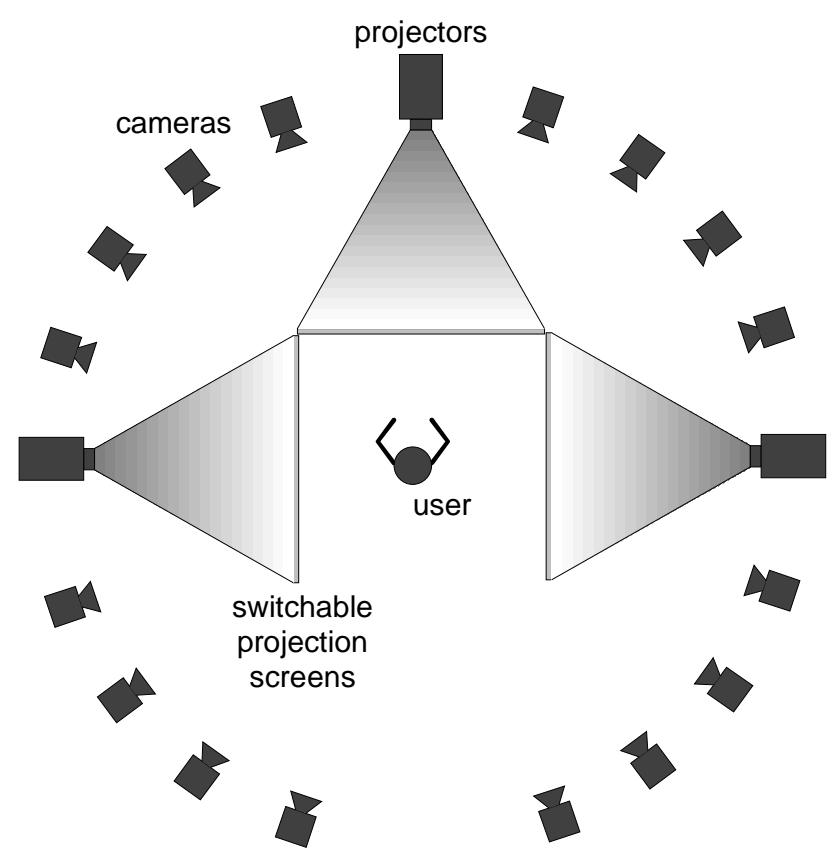

Figure 1. The blue-c configuration (top view)

The projection system is connected to a SGI Onyx3 with two graphic pipes, one with two display generators and one with four display generators. The cameras are connected to a PC cluster with 16 single processor nodes and two dual processor nodes. Each single processor node is connected to one camera over a fire wire cable.

\section{System decision}

Basic decisions have to be made at the very beginning. The first one is the shape of the projection screen and the room. This determines whether an edge blending or a predistortion of the projection is required. The second decision is on active or passive stereo projection. A passive, polarized system needs special and expensive screens, which keep the orientation of the polarization, while an active system needs expensive shutter glasses instead. The third decision is on the number of stereo projection units to be used. The overall system costs, the available computer power and the available space for the whole setup are the most important criteria for this decision.

The blue-c is a three sided projection system with three flat projection walls placed in a classic rectangular configuration. Each projection wall is made of three LCpanels $(950 \mathrm{~mm} \times 2240 \mathrm{~mm}, 80 \mathrm{~kg})$ with a $2 \mathrm{~mm}$ gap in between. This is due to the limited manufacturing capabilities of the distributor and due to the limited width of the integrated LC-film. This projection configuration gives good immersive results while keeping the technical complexity low. Furthermore the LC-panels are mostly available in a flat shape and with a maximum width of 950 $\mathrm{mm}$. A fourth projection on the floor would have further improved the immersion quality but would also have increased the complexity and the overall cost. Due to the limited space (height) in the room and the increasing costs the decision was made not to implement the projection on the floor.

\section{Mechanical construction}

In the beginning of the mechanical layout, a full list of all components to be integrated should be available. In our case this is the tracking system (Ascension Flock of Birds), 6 loudspeakers (Yamaha MSP5), 1 subwoofer (Yamaha SW10), active illumination, IR-emitters and active projection screens. The mechanical construction is shown in Figure 2.

Since a magnetical tracking system was chosen, the basis of the construction is a wooden platform with a total high of $540 \mathrm{~mm}$. The tracking emitter is integrated in the middle of the wooden platform and thus not visible to the user. The two lower front loudspeakers and the subwoofer are also integrated in the platform. The floor of the platform can be easily opened to access all the integrated components. The upper construction is realized with fiber reinforced composites (glass and carbon). They are stiff enough to keep the LC projection panels safely in place and at the same time still small and nice from a design point of view. Cable channels are integrated in the beams with enough space for the cables of all the components placed on the upper part of the construction, including the cables for the active projection screens. Together with the wooden platform there is enough space for all cables and connectors of the system. The chosen materials, wood and composite fibers, assure an accurate function of the tracking system. The cables as well as the loudspeakers, which are in a distance of $1.5 \mathrm{~m}$ to the emitter of the tracking system, do not have an important influence on the accuracy. The results from preliminary tests showed that the electrically switched glass panes do also not interfere with the tracking system. 


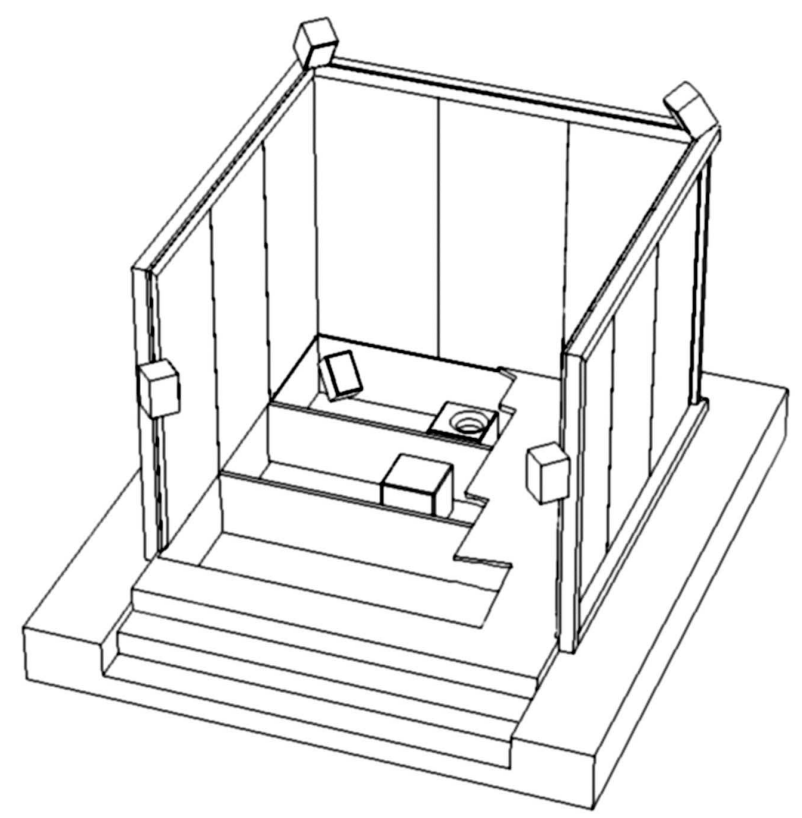

Figure 2. The mechanical construction of the blue-c

\section{Projection system}

The active LC-projection screens do not support passive stereo projection due to their depolarization properties. Therefore an active projection system was chosen for the blue-c. In the year 2000, when the design and the construction of the system was started, active stereo DLP-projectors were just emerging and still much too expensive for our budged. The state-of-the-art CRT projectors on the other hand where still quite expensive, compared to the light output. Furthermore, we were looking for a system which could be turned black during the camera acquisition period, therefore 60 times per second. The decision was taken to use two three chip LCD projectors (Sanyo XF12, 3500 ANSI lumen) per side and external LC shutters in front of the projection lens. With these shutters it is possible to generate an active stereo projection by alternating the output of the two projectors and block them both during the picture acquisition period (Fig. 3).

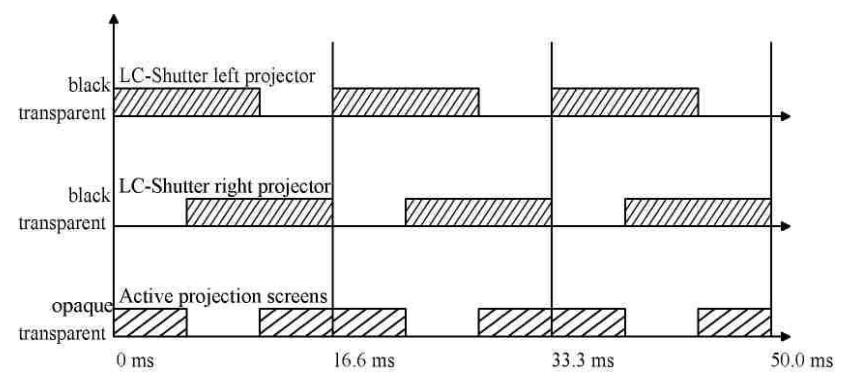

Figure 3. Timing diagram

The two projectors per side are mounted in a rack for a passive stereo projection installation (Fig. 4). The LCshutters are custom made ferro-electric liquid crystal shutters with an aperture size of $140 \mathrm{~mm} \times 140 \mathrm{~mm}$. The shutters are mounted in front of the 1:1.2 projection lenses. This lens allows to project directly on to the projection wall without any additional mirrors. Using no mirrors in the projection path eases the justification of the two LCD-projectors for active stereo projection. The shutters do not have to be synchronized with the Onyx and projectors, thus leaving complete freedom to the shutter times. This principle would not work with a single chip DLP projector, since the sequential generation of the three colors by a so-called color wheel would require a complicated synchronization of the shutters with the projectors.

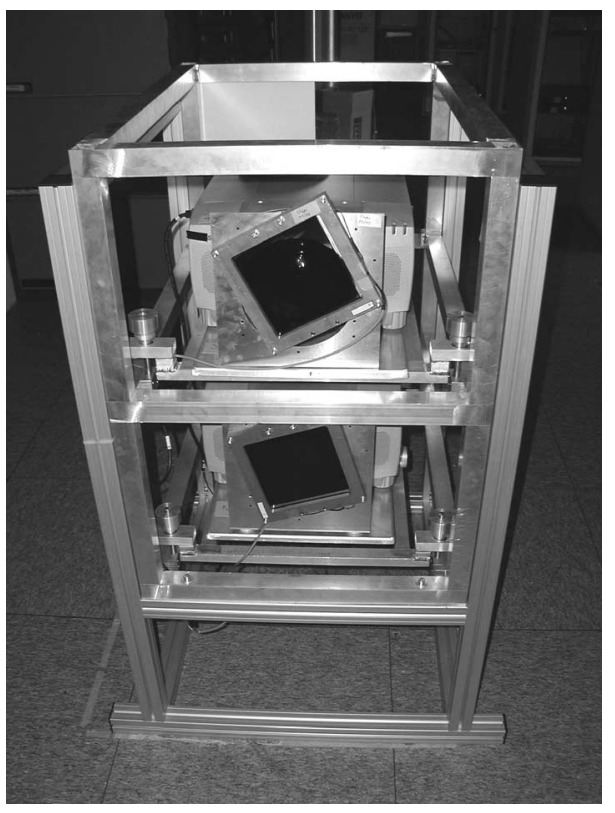

Figure 4. Stereo projection rack with LC-shutters 


\section{Connection of the components}

Figure 5 shows an overview of all components of the blue-c and the connection scheme. A total of 6 projectors and 4 monitors are connected to the SGI Onyx. The 16 cameras are connected with 16 fire wire cables to the computer cluster. The active projection panels, the 6 ferro-electric shutters in front of the projectors, the active illumination, the cameras as well as the IR-emitters are synchronized by a custom made control electronic.

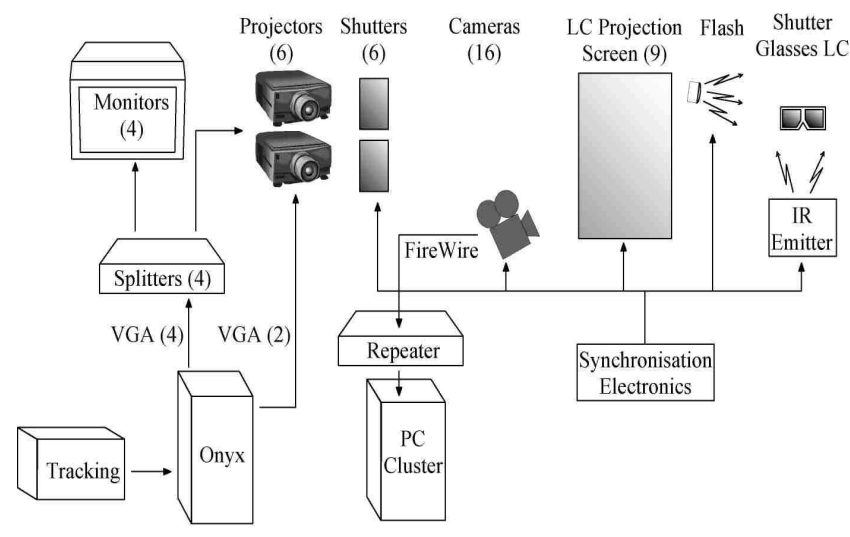

Figure 5. Component overview

As it can be seen clearly from this figure, there is no electrical connection between the Onyx (together with the projectors) and the rest of the technical installation

\section{Video cables and splitters}

The output from the SGI Onyx is a 13W3 connector, while the projector can accept the signal through a VGA or an coaxial RGBHV input. The best signal quality is achieved by using a $13 \mathrm{~W} 3$ to RGBHV adapter connected to 5 coaxial cables going to each of the projectors. This solution is rather expensive. In the blue-c the projectors are connected with inexpensive VGA cables over a 13W3 to VGA adapter to the Onyx. The VGA cables have single shielding for the three colors RGB and a length of $10 \mathrm{~m}$ and $20 \mathrm{~m}$ respectively, depending on the projector location. The signal going to the projectors is a XGA $(1024 \times 768)$ at $60 \mathrm{~Hz}$ signal. The $10 \mathrm{~m}$ cable does not show any attenuation of the signal. However, the $20 \mathrm{~m}$ cable shows a light shadow on the right edge of dark parts projected on a light background. This shadow is very light and barely noticeable. Independent of the cables, the three chip LCD projectors of the blue-c have a shift between the RGB colors of up to $1 / 2$ pixel. This is caused by a misalignment of the three chips and inherent for most of the 3-chip LC-projectors.

Up to five different kinds of $13 \mathrm{~W} 3$ to VGA or RGBHV adapters exist. In all cases the RGB signals are connected the same way, but the synchronization signals $\mathrm{H}$ and $\mathrm{V}$ unfortunately not. Therefore the popular Sun converters for example will just work if sync on green is used, which is not recommendable. The adapters should be checked very carefully to avoid any time consuming troubleshooting of signal problems later on.

The control room of the blue-c is equipped with four SGI monitors which share the graphic pipes with four of the six projectors. When an application is running in the blue-c, the operator can see both channels of the blue-c's middle projection screen as well as the two left eye channels of the left and right screens. When no application is running in the blue-c, the monitors can be used at full resolution of $1920 \times 1080$. Four two way splitters are connected to the corresponding outputs of the SGI. The splitters have a bandwidth of $500 \mathrm{MHz}$ (3dB bandwidth) and cost roughly US \$300.-. No attenuation of the signal could be noticed after adding the splitters. Some earlier tests with other splitters did not give the same positive results. The signal was attenuated by these splitters. This additional attenuation by the splitters resulted in a drastically darker projected image.

\section{Projector calibration}

Since flat screens are used, no pre-deformation of the image is needed and the calibration effort is reduced to a electronic and a mechanical calibration. Due to the rectangular shape of the blue-c no edge-blending between the three different screens is needed.

\subsection{Electronic calibration}

The calibration of the projectors concerns mainly the phase and sync settings. This implies that the SGI's graphic pipes are set to the projectors native resolution and frequency. The phase and sync values of the projectors are tuned so that each pixel of the SGI graphic pipe is mapped to the corresponding pixel of the projector. This calibration is done best with an appropriate static test image. The test image should contain a one pixel wide frame, surrounding the image and pixel alternating black and white pattern in the rest of the image. An experienced person needs approximately 20 min for the calibration of one projector. If a splitter is set in the path, the calibration has to be redone. 


\subsection{Mechanical calibration}

The electronic calibration of all six projectors is followed by the mechanical alignment of the projectors. This mechanical alignment is needed for an exact stereoscopic projection as well as for a sharp monoscopic image. A test pattern with a large mesh can be used for this purpose. The mesh should consist of one pixel wide lines.

Each projector rack of the blue-c has one projector mounted with 4 degrees of freedom and one with 6 degrees of freedom. The mechanical calibration in the blue-c is started with the center projector, 4 degrees of freedom. To adjust the missing two degrees of freedom, the whole projector stack can be moved around. After having aligned the first center projector, the image of the second projector has to be adjusted to fit exactly to the first one. The same procedure has to be repeated with the left and the right projector stack with the only difference, that they also have to be in line with the middle unit. A satisfying mechanical calibration takes about $4 \mathrm{~h}$.

\section{Cameras}

16 fire wire cameras are placed around the blue-c on a special aluminum construction. The construction is based on standard aluminum elements and offers a maximum flexibility to place cameras and other components. Each camera is connected via fire wire to one node of the PCcluster. The signal is amplified every 5 meters with a repeater. Up to 3 repeaters are used for each camera.

Dragonfly ${ }^{\mathrm{TM}}$ cameras since they offer a good sensitivity and a high picture acquisition frequency up to $15 \mathrm{~Hz}$. However, cameras with a even higher sensitivity would be useful in order to reduce the intensity of the flash illumination. On the other hand the picture acquisition frequency is high enough, since the bottleneck is in the processing of the acquired images. First tests showed, that a repetition frequency of $10 \mathrm{~Hz}$ seems to be sufficient for a collaborative work.

\section{Driver electronics and active projection screens}

The active projection screens are based on LC technology and can be switched to transparent by applying a voltage of $60 \mathrm{~V} \mathrm{AC} \mathrm{[9].} \mathrm{If} \mathrm{the} \mathrm{voltage} \mathrm{is} \mathrm{removed,} \mathrm{they}$ turn back to the original opaque state. The standard driver electronic from the LC-panel manufacturer is not optimized for fast switching between the two states. For the blue-c application, a custom made electronics was built. Furthermore, the electronics is used to synchronize and drive the other components of the blue-c. A PIC $16 \mathrm{~F} 877$ micro-controller is used to synchronize all the different events of the blue-c. Separate driver boards have been designed to drive the ferro-electric shutters in front of the projectors and the active projection screens. Standard 5 V TTL outputs are used to trigger the cameras, the active illumination and the IR-emitters for the shutter glasses.

The advantage of using a micro-controller is the flexibility in changing the timing and also the shape of all triggering signals for the components. This is of importance when a new device has to be integrated into the whole setup or an old device has to be replaced by a slightly different one.

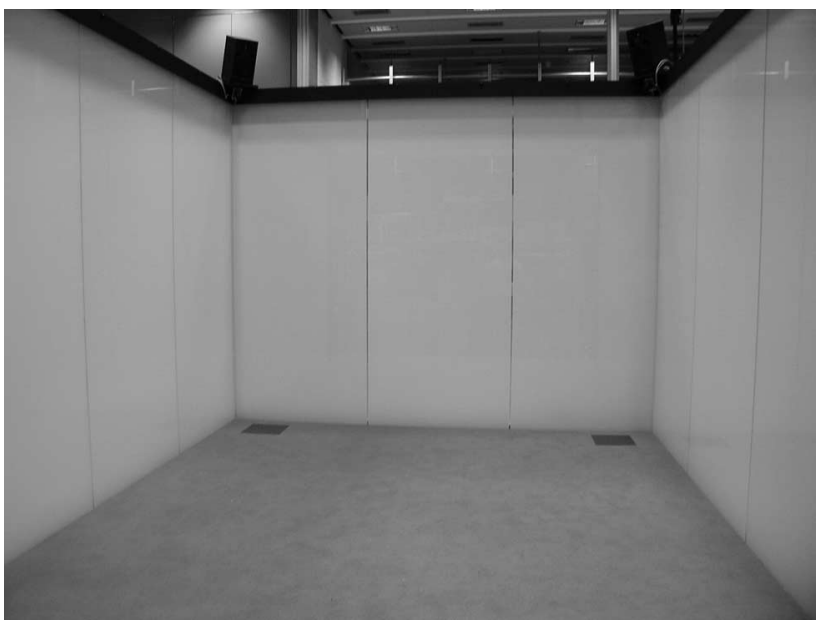

Figure 6. View inside the blue-c with opaque projection screens

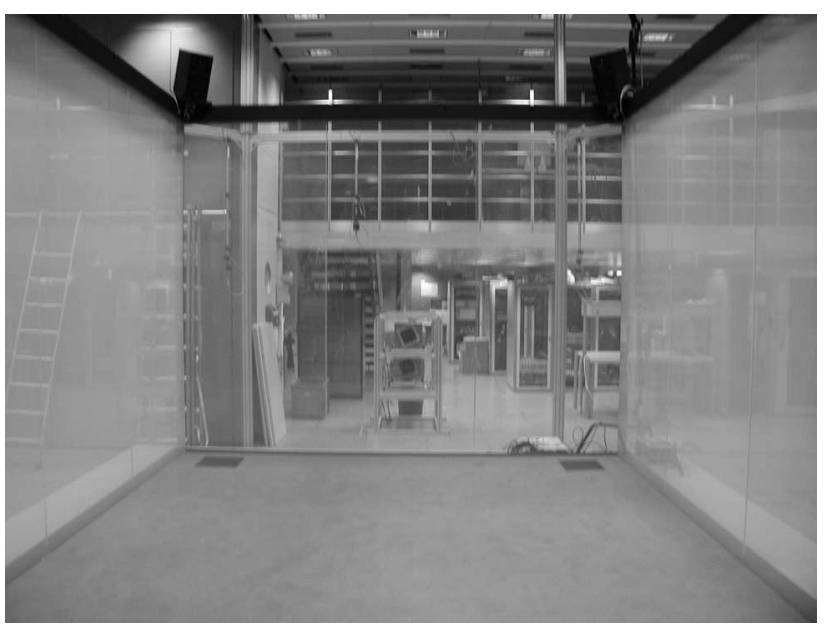

Figure 7. View inside the blue-c with transparent projection screens 


\section{Conclusions}

All triggered components, except for the cameras, are driven with a frequency of $60 \mathrm{~Hz}$. At this frequency the user does not notice any flickering of the active components. The cameras on the other hand are triggered with a frequency of $10 \mathrm{~Hz}$. A higher trigger frequency of the camera causes artifacts in the captured images.

The active projection screen is driven at its physical limits. The transition time from opaque to transparent (10\% to $90 \%$ ) takes approximately $2 \mathrm{~ms}$, while the transition from transparent to opaque (90\% to $10 \%$ ) takes approximately $6 \mathrm{~ms}$. The slow transition from transparent to opaque is given by the internal relaxation forces of the LC-film and can not be accelerated electrically. This nonideal behavior can be minimized by optimizing the time pattern of the different components [7]. For the user just the hotspot of the projectors gets a little more evident but remains at an acceptable level. The active projection screens offer a very brilliant and homogenous image.

The junctions between the three projection screen panels are visible but not disturbing. The LC film of the active projection panels is integrated between two glass panels. The surface of this glass panels is treated with an antireflective coating. Nevertheless some reflections from the other projection screens are evident. The reflection can be compared to the reflection on a CRT-TV in medium bight illuminated room. The reflection is noticeable when concentrating on it and disappears as soon as concentrating on the projected images. Since the projectors are darkened during the transparent state of the projection screens, no reflection disturbs the camera acquisition. The cameras are positioned in such a way they can see the whole body of the user, even if the user gets very close to the projection screens.

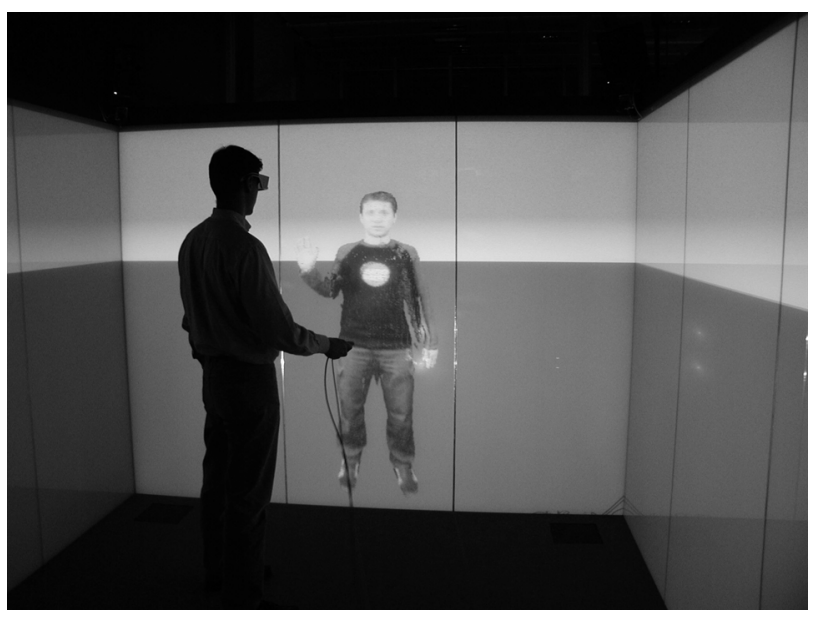

Figure 8. Collaboration in the blue-c
The channel separation of the stereoscopic projection is very good. The total performance of the projection is convincing. The brightness is higher compared to a traditional CRT stereo projectors at a lower price per stereo projection rack. Comparisons of the color fastness were made between the electrically switchable glass and

The six channel audio system showed to be very powerful. The user can precisely localize a simulated noise or sound source. The subwoofer located in the wooden platform is also important for the good audio performance. Furthermore it can transmit vibrations to the user at lower frequencies. The acoustic stimulus plays an important role for a high immersion.

The mechanical construction showed to fulfill the demanded requirements. Its stability and stiffness give a good sense of confidence in the blue-c. The user never gets uncomfortable about touching the projection screen or walking around, as he does in some other VR installations. The flexibility of placing new components and cables as well as the quick access to all the components proved to be a very valuable feature of the blue-c construction.

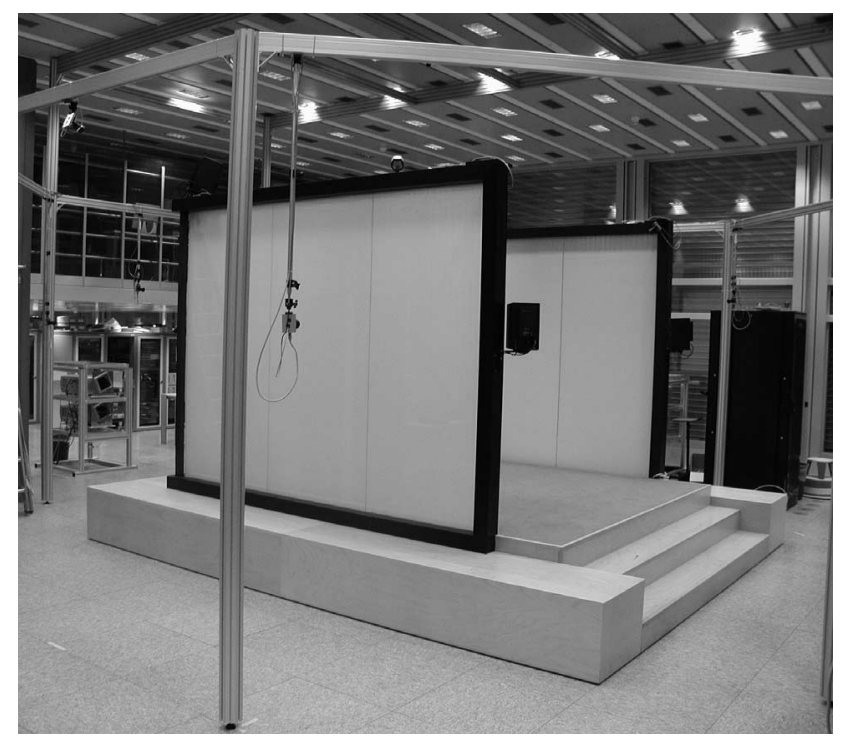

\section{Figure 9. Blue-c construction with camera mounting}

\section{Further work}

The weakest point of the actual blue-c is the active illumination with stroboscopic lights. The lights are very noisy and the light output is not constant between the different flashes due to variations in the light arc position of each discharge. Furthermore, the stroboscope also produces a high electromagnetic impulse that occasionally interferes with other electronic devices, depending on the distance between, e.g. with the sound system, where 
crackling noise is inducted. Another crucial factor is that the active shutter glasses are synchronized via a infrared link. Depending on the user's position in the blue-c this synchronization is disturbed by the stroboscope. An LED array promises to give better results since LED produce no noise and do not have an infrared part in their spectrum. Preliminary test showed that LED flashes do not interfere with the infrared link of the shutter glasses.

Further improvements can be expected from the active projection screens manufacturer, since they are not optimized for fast on-off cycles. Beside optimizing the material they also will be able to produce larger LCscreens and thus a whole wall could be manufactured as one piece. This will eliminate the gaps between the glass panes.

By the end of 2002 a second blue-c will be realized at the ETH Zurich that will allow to perform telecollaborative teamwork between the two stations. This installation will use an active 3-chip DLP projector, which allows to reduce significantly the time needed for the electrical and mechanical calibration.

The electrical implementation of the third step into the shutter glasses will be replaced by changing the program inside the microprocessor, which is integrated within the glasses. This will remove the cable which we use for the moment and allow the user to move freely within the bluec. The shutter glasses will also be modified with additional blenders to avoid irritation of the user by ambient light from the projection and from the flash illumination.

Further work will also use the camera signal to perform the tracking of the person. This will replace the magnetic tracking system that is in use now.

In a planned collaboration with universities from the United States it will be examined, how people can collaborate in such installations and how methods can be improved or changed to shorten development time and to reduce travel expenses by using this new technologies [10], [11], [14], [16].

\section{Acknowledgment}

This project was funded as an internal research project no. 0-23803-00 by the Swiss Federal Institute of Technology Zurich. We would like to thank the whole project team.

\section{References}

[1] C. Cruz-Neira, D.J. Sandin, and T.A. DeFanti, „Surroundscreen projection-based virtual reality: The design and implementation of the cave." Proceedings of SIGGRAPH 93, August 1993, pp 135-142.
[2] Kunz, A.; Spagno, C.: "Modified Shutter Glasses for Projection and Picture Acquisition in Virtual Environments"; IEEE Virtual Reality 2001 Conference, Yokohama, Japan; ISBN 0-7695-0948-7, ; March 2001, pp 281-282.

[3] Kunz, A.; Spagno, C.: "Novel Shutter Glass Control for Simultaneous Projection and Picture Acquisition" (PPT); Immersive Projection Technology and Virtual Environments 2001,; ISBN 3-211-83671-3, Springer-Verlag Wien/New York, Stuttgart (Germany), May 2001, pp. 257-266.

[4] Gross, M.; Kunz, A.; Meier, M.; Staadt, O.: "The Blue-C: Integrating Real Humans into a Networked Immersive Invironment"; CVE Conference 2000;

[5] Kunz, A., Spagno, C.; "Technical System for Collaborative Work“, Virtual Environments 2002, Barcelona (Spain), May 2002, pp. 73-80

[6] http://www.trimension-inc.com/index_reactor.html

[7] C. M. Lampert, "International Advances in Chromogenic Switching Technology," presented at Optical Materials Technology for Energy Efficiency and Solar Energy Conversion XV, San Diego, California, pp. 206-216, 1997.

[8] H. Fuchs, G. Bishop, K. Arthur, L. McMillan, R. Bajcsy, S. Lee, H. Farid, and T. Kanade. „Virtual space teleconferencing using a sea of cameras." Technical Report TR94-033, The University of North Carolina at Chapel Hill, Department of Computer Science, 1994.

[9] W. Elspass, L. VanGool, M. H. Gross, A. Kunz, M. Meier, G. Schmitt, O. Staadt, P. Stucki „The BlueCave"; ETHZinternal research proposal; Swiss Federal Institute of Technology (ETH) Zurich, Switzerland and University of Zurich, Switzerland

[10] T. Disz, M. Papka, M. Pellegrino, and R. Stevens. „Sharing visualization experiences among remote virtual environments." In Proceedings of the International Workshop on High Performance Computing for Computer Graphics and Visualization, July 1995

[11] V.D. Lehner and T.A. DeFanti. „Distributed virtual reality: supporting remote collaboration in vehicle design." Computer Graphics \& Applications, 17(2): 13-17, 1997.

[12] R. Raskar, G. Welch, M. Cutts, A. Lake; L. Stesin, and H. Fuchs. „The office of the future: A unified approach to imagebased modeling and spatially immersive displays." Proceedings of SIGGRAPH 98, pages 179-188, July 1998.

[13] Tetsuro Ogi, Toshio Yamada, Ken Tamagawa, Makoto Kano, Michitaka Hirose; "Immersive Te-lecommunication Using Stereo Video Avatar"; IEEE Virtual Reality 2001 Conference; March 13.-17. 2001; Yokohama, Japan; ISBN 07695-0948-7 
[14] Fuchs, D. K.; Lindemann, U.; Jokele, B.; Anderl, R.; Kleiner, S.; Fadel, G.; Greenstein, J.: „Sensitization to the Distributed Development Process"; Proceedings of the TMCE 2002; April 22.-26.; Wuhan, China

[15] Hirose, M.; Ogi, T.; Ishiwata, S.; Yamada, T.: "Development and Evaluation of the Immersive Multiscreen Display CABIN"; Systems and Computers in Japan, Vol. 30, No.1, pp.13-22, 1999

[16] Bailett, A.; Calahan, J.; McCluskey, S.: "Coordination at different stages of the product development process"; R\&D Management 28; 1998 